AperTO - Archivio Istituzionale Open Access dell'Università di Torino

La costruzione di un percorso educativo di empowering per giovani diabetici insulinodipendenti tipo 1

This is the author's manuscript

Original Citation:

Availability:

This version is available http://hdl.handle.net/2318/86536

since

Published version:

DOI:10.3280/PDS2011-002012

Terms of use:

Open Access

Anyone can freely access the full text of works made available as "Open Access". Works made available under a Creative Commons license can be used according to the terms and conditions of said license. Use of all other works requires consent of the right holder (author or publisher) if not exempted from copyright protection by the applicable law. 


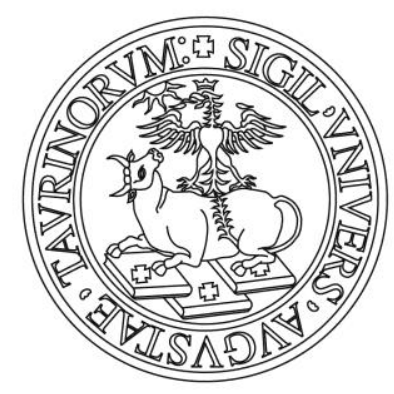

\section{UNIVERSITÀ DEGLI STUDI DI TORINO}

This is an author version of the contribution published on:

Questa è la versione dell'autore dell'opera:

Psicologia della Salute, 2, 201, DOI: 10.3280/PDS2011-002012

The definitive version is available at:

La versione definitiva è disponibile alla URL:

http://www.francoangeli.it/riviste/Scheda_Rivista.aspx?ID

Articolo $=42879 \&$ Tipo $=$ Articolo\%20PDF \&lingua $=i t \& i d R i$ vista $=72$ 


\title{
La costruzione di un percorso educativo di empowering per giovani diabetici insulinodipendenti tipo 1
}

\author{
Alessandra Mauri ***, Susanna Schmidt **, Antonella Scantamburlo \\ ***, Massimo Orrasch***, Tania Ciani***, Pierpaolo Faronato ****, \\ Agostino Paccagnella ***
}

\section{Riassunto}

Educare alla salute significa con Green (1990) "Ogni combinazione di esperienze di apprendimento che mirano a predisporre, facilitare e rinforzare gli adattamenti volontari del comportamento di salute individuale ". Gli Autori presentano il processo di costruzione e i dati preliminari del percorso educativo proposto a giovani diabetici insulino dipendenti di età compresa tra i 16 e i 26 anni afferenti al Servizio di Malattie del Metabolismo e Nutrizione dell'Ulss 9 di Treviso (MMN). L'approccio di cura al diabete viene rivisitato in un'ottica biopsicosociale e l'intervento educativo viene ristrutturato per passare da un modello di information giving e individuale ad un modello di empowering di gruppo con particolare attenzione allo sviluppo delle skills for life. Vengono presentate le fasi di trasformazione e costruzione del nuovo percorso educativo che ha coinvolto i diversi operatori del Servizio MMN. I dati clinici e comportamentali preliminari vengono discussi in funzione degli sviluppi futuri e della soddisfazione percepita.

Parole chiave: empowering, educazione terapeutica, diabete tipo 1

\section{Summary}

The construction of the educational intervention of group empowering for type 1 diabetes patiens

According to Green (1990) "health education" means "Every combination of experiences of learning that aim to predispose, to facilitate and to strengthen voluntary adaptations of behavior of individual health". The authors present the preliminary data of the intervention program proposed to young insulindependent diabetics aged between 16 and 26 years consulting the Service of Illnesses of the Metabolism and Nutrition (MMN) of the Ulss 9 of Treviso. The approach of care to diabetes is proposed in a biopsicosocial optic and the educational intervention is restructured by passing from an informationgiving model to a model of group empowering with particular attention to the development of the life skills. The phases of transformation and construction of the new educational program which involved different operators of the Service MMN are introduced. The preliminary clinical and behavioral data are discussed in the optic of future developments and of perceived satisfaction.

Key words: empowering, terapeutic education, diabetes type 1 


\section{Introduzione}

\section{La malattia cronica}

Vi sono alcune malattie oggi curabili dalla medicina che non sono però guaribili. Ciò rappresenta, per coloro i quali ne sono affetti, l'ingresso in una condizione particolare qual'è quella della malattia cronica. A livello medico la capacità di identificare la malattia permette di definire la diagnosi e di prescrivere il giusto trattamento. Tale iter è spesso possibile e sufficiente nella fase acuta della malattia, o in stati acuti in generale, dove il bisogno è prevalentemente biomedico e l'intervento terapeutico che segue la diagnosi è definibile nel tempo. La definitezza del tempo e del tipo di intervento necessario per ottenere la guarigione, anche in casi gravi o di terapie invasive, favorisce la "compliance" e l'aderenza al trattamento e promuove la motivazione del paziente alla cura e alle prescrizioni necessarie per la terapia (assumere farmaci, gestire presidi, ecc). Molto diversa è la diagnosi di una malattia che ha carattere cronico, da cui il paziente non guarisce, e che necessita di trattamento continuativo, prolungato per tutta la vita e fondamentale sia per la sopravvivenza che per evitare complicanze e aggravamento dello stato di salute. Per questo motivo nelle malattie croniche la diagnosi e i conseguenti interventi educativi legati alla gestione delle terapie sono da considerarsi un momento estremamente delicato a cui vanno dedicati tempo e attenzione particolare (Lacroix e Assal, 2005). In questo caso il solo approccio biomedico è insufficiente, poiché sono molteplici gli aspetti in gioco: le aspettative, i bisogni, le rappresentazioni del paziente, le fasi della crescita, il momento della diagnosi e molte altre variabili ancora.

Il diabete mellito di Tipo 1 rappresenta una condizione clinica importante per la valutazione di un modello di intervento educativo adeguato alla gestione delle malattie croniche.

\section{Modelli di educazione terapeutica e diabete}

La terapia del diabete richiede da parte del paziente un'assunzione di responsabilità che favorisca l'attivazione di stili di vita adeguati alla gestione della malattia. Indicativa a riguardo è l'osservazione di Funnell e Mensing (2005): «Pensavamo che il nostro lavoro fosse di 
far fare alle persone ciò che avrebbero dovuto fare e ci siamo accorti che tutto questo non funzionava: le persone inglobavano il diabete nella loro vita piuttosto che cambiare qualcosa della vita a causa del diabete». L'educazione terapeutica è un importante strumento per favorire l'attivazione di questa consapevolezza. È da tempo segnalata la necessità di un approccio educativo (Assal e Lacroix 2000; Zoffmann e Lauritzen, 2006; Gentili e Gentile, 2010) che preveda un'educazione di tipo non tradizionale o di sola informazione, tipica del modello biomedico, ma più adeguata a contesti di malattia acuta, e che introduca spazi educazionali e di sostegno in un'ottica biopsicosociale. L'intervento di empowering nell'educazione del soggetto diabetico insulinodipendente si fonda su un'azione educativa differente, il cui obiettivo è quello di rendere i soggetti capaci, autonomi e autosufficienti (Miselli, 2006). L'operatore ha funzione di supporto nel processo di apprendimento finalizzato ad una gestione autonoma della propria salute. Nodo centrale dell'empowerment è formare persone "potenti" per favorire l'adozione di comportamenti di salute (Dallago, 2006).

\section{Il modello skills for life applicato al diabete}

L'intervento di promozione del benessere in soggetti con il diabete di tipo 1 e qui presentato, è stato progettato attraverso la creazione di un percorso di educazione di gruppo, rivolto a giovani diabeticiinsulino dipendenti e con riferimento al modello proposto dalla Organizzazione Mondiale della Sanità (1992) della life skills education.. In tale modello, le abilità di vita sono descritte come quelle «Capacità di adattamento e di comportamento positivo che permettono agli individui di affrontare in maniera efficace le esigenze e le sfide della vita quotidiana. Abilità personali, interpersonali, cognitive e fisiche che consentono alle persone sia di controllare e orientare la propria vita, sia di sviluppare la capacità di vivere con, e di produrre cambiamenti nel proprio ambiente». L'acquisizione delle diverse competenze necessarie ad una buona gestione del diabete è strettamente correlata all'attivazione di un adeguato stile di vita che comprende una corretta alimentazione, lo svolgimento di attività fisica regolare, l'automonitoraggio glicemico e la efficace somministrazione della terapia. Ma nella gestione della malattia vanno considerate anche le emozioni relative alla propria condizione di cronicità, l'acquisizione di competenze cognitive (ad esempio le abilità di problem solving o di 
ristrutturazione cognitiva), le abilità di gestione delle emozioni e le competenze sociali. Il modello educativo skills for life ben si presta come modello di riferimento per gli interventi con bambini e adolescenti e giovani adulti con diabete insulinodipendente. Il riferimento a tale modello mira alla costruzione di spazi educativi ove potenziare le abilità necessarie ad affrontare la vita e la gestione del diabete e ha lo scopo di attivare ed ottimizzare i comportamenti e gli stili di vita adeguati. In tale ottica un intervento educativo per soggetti diabetici, oltre a sviluppare le specifiche competenze che debbono essere acquisite per la gestione del diabete, dovrebbe abilitare i soggetti a gestire diverse problematiche quotidiane (prendere decisioni, risolvere problemi, trovare soluzioni creative, analizzare e valutare situazioni) e padroneggiare le relazioni (abilità relazionali e sociali, comunicazione interpersonale).

\section{L'esperienza nell'Unità Operativa di Treviso}

\section{Il background}

Per meglio comprendere le cause e le finalità che hanno promosso il progetto di seguito descritto è necessario introdurre due brevi note "storiche". L'ambulatorio dell'U.O. di Malattie Metaboliche e Nutrizione Clinica dell'ULSS 9 di Treviso proponeva un approccio di cura di tipo direttivo/prescrittivo, privilegiando il modello educativo di information giving. La visione della cura coincideva prevalentemente con il "fornire informazioni" specifiche al paziente, aggiornate e dettagliate, relative alla terapia e ad eventuali presidi. In questo contesto il paziente veniva "seguito nel tempo" per dirigerlo verso comportamenti di automonitoraggio e obiettivi di autocura predefiniti. Lo spazio per il paziente era solo individuale e di visita in ambulatorio con lo specialista, preferibilmente medico. Il secondo elemento degno di nota è legato al fatto che, fino all'inizio del progetto attuale, non era mai stata misurata la compliance dei pazienti, e noon si era mai posta attenzione al fatto che vi fosse un cospicuo numero di soggetti con diabete non compensato (livelli di emoglobina glicata al di sopra della norma).

Il presente progetto è stato pensato anche per gli operatori del servizio, per offrire una nuova opportunità di valutare il concetto di cura, ed in particolare di cura di malattie croniche, e inserire una nuova 
direzione che cogliesse i problemi in maniera diversa e proponesse nuove soluzioni.

\section{Empowering di gruppo: la nuova proposta}

Muovendo da tali presupposti, si è deciso di dare vita al presente progetto scegliendo di lavorare con pazienti con diabete di tipo 1 che, per i motivi descritti nel precedente paragrafo, pur presentando una malattia fortemente a rischio di complicanze (retinopatia diabetica, insufficienza renale cronica, patologie cardiovascolari di vario livello, ecc), non avevano ricevuto tutte le attenzioni e le opportunità educative e terapeutiche precedentemente citate. Con il presente progetto si è andati a modificare la normale strutturazione delle visite specialistiche diabetologiche presso l'ambulatorio MMN. La routinaria visita ambulatoriale normalmente svolta dal medico diabetologo con un approccio di tipo "frontale" è stata sostituita da uno spazio di intervento educativo di gruppo, considerando anche altri modelli di interventi educativi proposti con diabetici di tipo 2 (Porta e Trento, 2004) e i modelli di sviluppo delle life skills (progetto educativo LSE; Marmocchi, 2004).

Gli obiettivi di questo nuovo spazio erano due:

a) favorire il ritorno di pazienti giovani adulti che non frequentavano più il centro per le normali visite di controllo;

b) proporre un nuovo spazio educativo per favorire nei pazienti lo sviluppo di comportamenti e stili di vita adeguati all'autogestione del diabete, considerando che più del $98 \%$ della cura del diabete viene fornita dal paziente stesso (Anderson e Funnel. 2002).

\section{FASE UNO La formazione degli operatori}

L'intervento educativo dedicato ai pazienti è stato preceduto da un periodo di formazione rivolto agli operatori del servizio MMN (dietisti, infermieri professionali, medici diabetologi) e di costruzione di un gruppo di lavoro multidisciplinare. La psicologa dell'MMN ha progettato e condotto gli incontri con i medici diabetologi, le infermiere e le dietiste sulle tematiche della metodologia educativa, delle dinamiche di gruppo, del modello di Empowering $\mathrm{e}$ dell'approccio alle Life Skills Education. Dopo la fase iniziale di apprendimento e formazione, gli operatori hanno lavorato insieme per 
costruire la struttura degli incontri coordinando le diverse funzioni e intersecando ruoli e competenze.

FASE DUE: la proposta ai pazienti

Il gruppo di partecipanti: gruppo di lavoro e gruppo di controllo

Il nuovo percorso di educazione terapeutica è stato proposto ai giovani pazienti con diabete mellito insulino-dipendente tipo 1 che accedono all'MMN di Treviso. 40 pazienti (età media $=20.6, D S=$ 2,7 , range 16-26) hanno risposto alla proposta partecipando al primo incontro informativo. Di questi 40, 27 hanno accettato di partecipare al percorso di formazione (e verranno d'ora in poi definiti gruppo di lavoro). I pazienti che hanno partecipato al primo incontro informativo, ma che, per vari motivi, non hanno aderito al percorso sono stati considerati come gruppo di controllo $(\mathrm{N}=13)$.

Il gruppo totale di partecipanti a cui ci si riferisce è quindi costituito da 40 giovani diabetici di cui $25(62,5 \%)$ donne e $15(37,5 \%)$ uomini. L'anno di esordio del diabete è dal 1989 al 2009 (range: 1-21 anni, $M$ $=10,95, D S=5,42)$. Tra il gruppo di lavoro e il gruppo di controllo non vi sono differenze nella distribuzione di genere, $X^{2}(\mathrm{~N}=40,1)=$ $.01, p=.931$ (Lavoro: 17 femmine, 10 maschi, Controllo: 8 femmine, 5 maschi), né per l'età, $F(1,39)=.70, p=.407$ (Lavoro: $M=20,44 ; D S=$ 2,89; Controllo: $M=21,23 ; D S=2,52)$, né per il numero di anni di diabete, $F(1,39)=.52, p=.475$ (Lavoro: $M=10,52 ; D S=5,39$; Controllo: $M=11,85 ; D S=5,58)$.

\section{FASE TRE: Analisi dei bisogni}

In un intervento di educazione terapeutica, l'analisi dei bisogni educativi è certamente un momento strategico importante. Nella valutazione dei bisogni del nostro gruppo di lavoro, la compliance è misurata con il numero di visite annue, la conoscenza con un questionario specifico e i vissuti emotivi relativi alla malattia con la rilevazione di sintomi e segni di burnout da diabete. Inoltre sono stati valutati i comportamenti di autogestione attraverso la rilevazione del il numero di automonitoraggi, i valori di glicemia media per periodi di 
riferimento definiti e la misurazione di alcuni comportamenti specifici di autogestione attesi.

\section{Rilevazione del numero di visite}

Ogni visita del paziente è registrata nella cartella computerizzata. Il numero di visite consigliato per il monitoraggio clinico del diabete è di tre-quattro visite annue.

\section{Valutazione delle competenze relative al sapere}

Un primo passo per la costruzione del nuovo intervento educativo è stato quello di valutare gli eventuali bisogni di conoscenza attraverso la somministrazione del Questionario sulla Conoscenza del Diabete, GISED 2001 (Vespasiani, 2002), che valuta le conoscenze e alcuni comportamenti del soggetto diabetico nella gestione della malattia. In particolare vengono valutate le conoscenze relative all'alimentazione, ai comportamenti di autocontrollo, alla gestione della glicemia, della ipoglicemia e dello scompenso glicemico, alla gestione della terapia insulinica e dell'attività fisica in relazione alla terapia. I dati rilevati permettono la diagnosi educativa e la ?costruzione degli incontri in funzione dei bisogni di empowering rilevati. Le attività di educazione del nuovo progetto di gruppo sono quindi state strutturate tenendo in considerazione sia le lacune conoscitive che le difficoltà relative all'attivazione dei comportamenti efficaci alla gestione terapeutica (ad es. comportamenti di gestione della ipoglicemia). Altro obiettivo delle attività proposte negli incontri di gruppo è quello di permettere la rilevazione degli eventuali problemi che ostacolano tali comportamenti e di ricercare le possibili soluzioni da attivare nella propria quotidianità. Il dato relativo al questionario Gised qui presentato riguarda i 27 soggetti che hanno partecipato al percorso.

\section{Valutazione dei disagi causati dalla malattia: il burnout da diabete}

Un'altra misurazione riguarda il numero di soggetti in burnout da diabete e i relativi livelli di burnout. Non esiste in letteratura un test specifico atto a misurare le dimensioni del burnout da diabete, ma Polonsky (2002) indica una serie di fattori e di comportamenti, che definisce sintomi, la cui rilevazione può aiutare a misurare tale 
dimensione nei pazienti con diabete. Nel nostro studio è stata creata una scheda di osservazione-rilevazione di tali sintomi (Tabella 1) ed è stata attivata una osservazione partecipata da parte di tre diversi operatori, durante gli incontri di gruppo e/o i colloqui individuali. Sono poi stati assegnati punteggi in funzione delle osservazioni fatte, e valutati i livelli di burnout dei nostri pazienti. Assegnando un punto ad ogni item, il punteggio totale è di 14 (presenza di burnout elevato), un punteggio da 7 a 13 è stato considerato di presenza di burnout di grado medio, un punteggio inferiore a 7 di disagio lieve o assenza di burnout. Essendo tale valutazione possibile solo osservando o intervistando i soggetti, è stato possibile condurre tale osservazione solo sul gruppo di lavoro.

Tabella 1. Scheda di osservazione dei sintomi e disagi espressi per la definizione del burnout. Adattata da Polonsky (2002).

\begin{tabular}{|ll|}
\hline $\begin{array}{l}\text { Scheda di osservazione per la valutazione del } \\
\text { Burnout da diabete }\end{array}$ \\
\hline 1. & esprime la sensazione che il D. stia prendendo il controllo della sua vita \\
\hline 2. & racconta di sentirsi oppresso dagli obiettivi \\
\hline 3. & racconta di sentirsi oppresso dalle azioni di cura di sé \\
\hline 4. & racconta di avere la sensazione di fallire con il diabete \\
\hline 5. & esprime forti sentimenti negativi sul diabete (rabbia tristezza frustrazione) \\
\hline 6. & esprime la sensazione di sentirsi solo e incompreso \\
\hline 7. & riconosce una cura di sé cronicamente scarsa e un controllo glicemico \\
\hline 8. & ncadente ha alcuna registrazione (o scarsa) di una cura regolare e continuativa \\
\hline 9. & esprime una forte ambivalenza nel migliorare la cura di sé \\
\hline 10. & può sentire che non vale la pena di impegnarsi nell'autogestione \\
\hline 11. & manifesta di sentirsi colpevole e/o spaventato per la scarsa cura di sé \\
\hline 12. & manifesta negazione rispetto ai problemi che ha nell'autogestione \\
\hline 13. & racconta di non farsi mai controlli \\
\hline 14. & racconta di non fare per periodi prolungati la terapia \\
\hline
\end{tabular}

\section{Valutazione dei comportamenti di autocontrollo}

Ai pazienti diabetici è richiesto in particolare di monitorare l'andamento glicemico ripetendo l'automonitoraggio più volte nella giornata, ciò per poter valutare le variazioni glicemiche ed effettuare adeguate correzioni della terapia. Le valutazioni della glicemia fatte, 
vengono memorizzate dal glucometro e i dati possono essere scaricati su un software dedicato all'interno della cartella clinica computerizzata. Tale comportamento di autocontrollo è considerato una condizione necessaria e fondamentale per la gestione della terapia e può essere valutato periodicamente con l'analisi dei dati registrati nella cartella computerizzata. Prima di ogni incontro i dati di ogni soggetto venivano scaricati in cartella. Era poi possibile utilizzare tali dati durante l'incontro di gruppo per discuterli e valutarli con tutti i partecipanti.

\section{Costruzione di obiettivi di cambiamento personali}

Nella prima fase del percorso sono stati considerati gli obiettivi relativi ai seguenti comportamenti di autogestione:

- automonitoraggio: aumento del numero di misurazioni

- gestione ipoglicemie: applicazione della regola del 15 (assunzione di 15 grammi di $\mathrm{CHO}$ ogni 15 minuti in presenza di ipoglicemia)

- "kit ipo": capacità di portarsi il quantitativo adeguato di zuccheri e di utilizzarlo per la gestione di ipoglicemie

- conta dei carboidrati $(\mathrm{CHO})$ : capacità di valutare la presenza di carboidrati semplici e complessi negli alimenti e di rispondere in modo adeguato con la terapia insulinica

- rilevazione e gestione delle lipodistrofie

- uso di ago adeguato alla terapia

- cambio dell'ago ad ogni iniezione (per evitare lipodistrofie)

La presenza o meno di ogni comportamento è stato rilevato attraverso due schede; una scheda di autovalutazione, costruita ad hoc in cui i soggetti valutavano e dichiaravano di avere o meno raggiunto l'obbiettivo di autogestione, e una scheda compilata dagli operatori durante gli incontri e durante le discussioni con i partecipanti, dove veniva registrata la presenza di comportamenti consoni agli obiettivi valutati attraverso giochi di ruolo e simulate.

Nel lavoro specifico relativo ai comportamenti di gestione di una malattia cronica è però impossibile omologare del tutto gli obiettivi tra soggetti diversi e diventa necessario dare spazio alla costruzione di obiettivi personalizzati che ogni soggetto si autodefinisce e che possono poi essere monitorati e discussi nel gruppo di lavoro. Per cui oltre agli obiettivi generali descritti sono stati anche proposti obiettivi 
personalizzati e individuali in funzione dei bisogni soggettivi. Tali obiettivi personalizzati venivano discussi di volta in volta nel gruppo insieme agli operatori, registrati in cartella per essere monitorati agli incontri successivi. Tutti gli obiettivi proposti utilizzavano il criterio di "obiettivo SMART" (specifico, misurabile, attuabile, realizzabile e temporalmente definito).

\section{La strutturazione degli incontri}

Ogni incontro prevedeva l'individuazione di un tema che guidava le attività e che veniva proposto con modalità diversificate. Ampio spazio è stato dato all'attivazione o riattivazione dei comportamenti più efficaci per l'autogestione del diabete, dati precedentemente e erroneamente per scontati nell'approccio individuale. I temi prevalentemente proposti erano stati scelti in funzione dei bisogni rilevati e riguardavano la gestione delle ipoglicemie e iperglicemie e i comportamenti di gestione delle tecniche di iniezione. Nel periodo di tempo descritto nel presente lavoro si è lavorato in particolare sull'autocontrollo e sulla gestione delle terapie insuliniche, sull'alimentazione, sull'attività fisica, sulla gestione delle ipoglicemie (la "regola del 15" e del "kit ipo), sulla gestione delle iperglicemie con le formule di correzione, sulle tecniche di best practice per le iniezione e sulla "conta dei carboidrati.

Gli incontri di gruppo si sono svolti ogni tre mesi circa per un totale di 4 incontri annui. Dal Maggio 2009 a Dicembre 2010 si sono svolti 7 incontri. Dei sei operatori coinvolti nel progetto, quattro partecipavano ad ogni incontro (un medico diabetologo, una dietista, un infermiere e la psicologa); l'incontro era cogestito ma condotto principalmente da uno degli operatori in funzione del tema trattato. I temi trattati erano sviluppati attraverso lezioni frontali interattive, analisi in gruppo dello scarico dati e ricerca delle cause dei problemi, simulate di problem solving, brainstorming di possibili soluzioni per i problemi proposti, schede di autovalutazione e di definizione di obiettivi specifici personalizzati.

\section{La valutazione soggettiva del nuovo modello proposto}

Per valutare la percezione soggettiva del cambiamento della tipologia di visita è stato costruito e somministrato sia ai partecipanti 
che agli operatori un questionario di valutazione, utile per individuare i punti di forza e di debolezza del nuovo modello proposto e per mettere a confronto la modalità di visita precedente, ambulatoriale e individuale, con la nuova modalità di empowering di gruppo proposta.

\section{Risultati preliminari}

Vengono presentati i risultati preliminari relativi alle misurazioni fatte sui partecipanti al gruppo di lavoro $(n=27)$. In alcune occasioni è stato possibile confrontare i dati con il gruppo di controllo (ove specificato) o effettuare misurazioni a distanza di un anno. Molti sono i dati mancanti relativi al gruppo di controllo a causa dell'alta percentuale di drop out (la misurazione e il confronto è stato possibile solo in 7 dei 13 partecipanti inizialmente destinati al gruppo di controllo).

\section{Numero di visite}

Riguardo al numero di visite effettuate, sono stati confrontati i dati dei due gruppi relativi a due periodi: trimestre precedente il primo incontro di gruppo, e il trimestre successivo al settimo incontro. Come si vede dalla Tabella 2, a livello descrittivo, nei due tempi, quelli del gruppo di controllo fanno meno visite; e mentre per il gruppo di lavoro c'è un aumento delle visite nel tempo, per il gruppo di controllo c'è una diminuzione. Al fine di determinare se queste differenze tra i due gruppi e tra $\mathrm{i}$ due tempi sono significative, è stata calcolata una MANOVA a misure ripetute con tempo come variabile entro soggetti e gruppo come fattore fra soggetti. Da questa analisi risulta che non c'è un effetto significativo del tempo $[F(1,36)=0,01, p=.914]$, mentre c'è un effetto significativo del gruppo $[F(1,36)=17,88, p<.001]$, e un'interazione tempo x gruppo, $F(1,36)=6,15, p<.05$. In particolare, i partecipanti del gruppo di lavoro effettuano più visite rispetto a quelli del gruppo di controllo, e il numero di visite aumenta col tempo per il gruppo di lavoro, mentre diminuisce per il gruppo di controllo.

Tabella 2: Confronto del numero di visite tra l'inizio e la fine del progetto, sia del gruppo di controllo che del gruppo di lavoro.

\begin{tabular}{ccll}
\multicolumn{1}{l}{ Gruppo Controllo $(n=13)$} & & \multicolumn{2}{l}{ Gruppo di Lavoro $(n=25)$} \\
& $D S$ & & $M$
\end{tabular}




\begin{tabular}{lllll}
\hline $\begin{array}{l}\text { Pre } \\
\text { incontri }\end{array}$ & 2,23 & 1,36 & 2,76 & 1,23 \\
$\begin{array}{l}\text { Fine } \\
\text { incontri }\end{array}$ & 1,46 & 1,13 & 3,60 & 1,47 \\
\hline
\end{tabular}

\section{Sapere vs. saper fare}

Le maggiori lacune conoscitive individuate sono relative a temi quali indici glicemici al risveglio, glicosuria ed emoglobina glicosilata, la funzionalità dell'insulina e le tecniche di conservazione dell'insulina. Anche se quasi il $70 \%$ dei pazienti dà risposte corrette relative ai comportamenti di autocontrollo, alcuni comportamenti auspicabili non vengono poi attivati. Nello specifico il $44 \%$ dei pazienti dichiara di non segnare mai i dati sul diario e il $90 \%$ non analizza mai i dati registrati; il $20 \%$ dichiara di non eseguire l'autocontrollo o di eseguirlo solo 1 volta al giorno (30\%). In generale $\mathrm{i}$ dati relativi ai bisogni di conoscenza mostrano che circa 1'85\% dei pazienti ha conoscenze adeguate relative alla malattia diabetica, in particolare sulla gestione di scompensi glicemici, sulla gestione della terapia insulinica (Tabella 3), ma solo il $30 \%$ è in grado di attuare un adeguato comportamento di gestione delle ipoglicemie, conoscere le formule di correzione delle ipoglicemie (regola del 15) e sa aspettare i tempi di risposta fisiologica senza ipercorreggere. In ulteriore contrasto con le conoscenze corrette (Sapere), i dati clinici (Saper Fare) mostrano una percentuale alta di pazienti con diabete non compensato: emoglobina glicosilata: $M=$ 8,$70 ; D S=1,74$; range: $6-14$.(valori di soglia $6 \%$ ). Non c'è differenza tra i due gruppi circa gli indici di emoglobina $F(1,34)=.722, p=.401$ (Lavoro: $M=8,52 ; D S=1,30$; Controllo: $M=9,04 ; D S=2,43$ ).

Tab 3. risultati in percentuale di risposte corrette al test di valutazione delle conoscenze relative al diabete e alla sua gestione GISED 2001 (Vespasiani, 2002.)

\begin{tabular}{|l|l|}
\hline Conoscenze relative a & \% di Risposte Corrette \\
\hline $\begin{array}{l}\text { Alimenti e gestione della } \\
\text { terapia }\end{array}$ & 65.05 \\
\hline $\begin{array}{l}\text { Comportamenti di } \\
\text { autocontrollo }\end{array}$ & 68.17 \\
\hline La malattia diabetica delle & 84.14 \\
\hline $\begin{array}{l}\text { Gestione di } \\
\text { ipoglicemie e terapia } \\
\text { scompensi glicemici }\end{array}$ & 86.27 \\
\hline Gestione to.24 \\
\hline
\end{tabular}




\begin{tabular}{|l|l|}
\hline insulinica & \\
\hline Attività fisica e terapia & 69.60 \\
\hline
\end{tabular}

\section{Burnout da diabete}

La rilevazione della presenza di sintomi di burnout è possibile solo attraverso interviste e osservazioni dirette, e i risultati sono presentati solo per i partecipanti del gruppo di lavoro. Essi mostrano un'elevata percentuale di disagio causato dalla malattia misurato con la scheda di osservazione partecipata. In particolare ben il $79 \%$ dei soggetti del nostro gruppo di lavoro manifesta presenza di sintomi (con punteggio alto e medio).

\section{Comportamenti di autocontrollo}

\section{Automonitoraggi e andamento glicemico}

Le analisi relative all'automonitoraggio e all'andamento glicemico mettono a confronto i due gruppi nei due tempi. Come riportato in Tabella 4, entrambi i gruppi hanno incrementato il numero di autocontrolli glicemici, con un aumento più pronunciato per il gruppo di lavoro. Per determinare se queste differenze tra i due tempi e i due gruppi sono significative, è stata calcolata una MANOVA a misure ripetute con tempo come variabile entro soggetti e gruppo come fattore fra soggetti. Da questa analisi risulta un effetto significativo del tempo, $F(1,28)=10,95, p<.01$, mentre l'effetto del gruppo, $F(1,28)=0,29, p$ $=.589$, e l'interazione tempo x gruppo, $F(1,28)=0,82, p=.374$, non sono significativi.

L'analisi dello scarico dei dati del glucometro di un singolo soggetto permette di misurare anche la glicemia media del periodo e la deviazione standard. ̇̀ possibile, inoltre, conoscere il numero di ipoglicemie rilevate durante il periodo osservato. La Tabella 5 riporta le statistiche descrittive relative a queste misure fisiologiche. A livello descrittivo, i risultati suggeriscono che la glicemia media diminuiva per ambedue i gruppi, ma sembra diminuire di più per il gruppo di controllo. Anche la deviazione standard diminuisce tra il periodo prima degli incontri e l'ultimo trimestre 2010 per ambedue i gruppi. Infine il numero di ipoglicemie analogo tra i due gruppi prima dello studio risulta oggi aumentato per il gruppo di lavoro. Queste differenze tra $\mathrm{i}$ 
due tempi e i due gruppi non risultano però significative [tempo, $F(3,23)=2,79, p=.063$; tempo $\mathrm{x}$ gruppo, $F(3,23)=1,09, p=.372$; gruppo, $F(3,23)=1,77, p=.182]$.

Tabella 4.Numero di letture effettuate nel trimestre precedente allo studio rispetto all 'ultimo trimestre 2010. Confronto tra gruppo di controllo e gruppo di lavoro.

\begin{tabular}{|c|c|c|c|c|}
\hline & \multicolumn{2}{|c|}{ Gruppo Controllo $(n=7)$} & \multicolumn{2}{|c|}{ Gruppo di Lavoro $(n=23)$} \\
\hline & $M$ & $D S$ & $M$ & $D S$ \\
\hline Pre incontri & 160,57 & 130.60 & 160,13 & 98,65 \\
\hline $\begin{array}{l}\text { Fine } \\
\text { incontri }\end{array}$ & 233,87 & 124,29 & 288,39 & 165,68 \\
\hline
\end{tabular}

Tabella 5. Risultati relativi alle misure fisiologiche effettuate nel trimestre precedente allo studio rispetto all'ultimo trimestre 2010. nei due gruppi

\begin{tabular}{|c|c|c|c|c|}
\hline & \multicolumn{2}{|c|}{$\begin{array}{l}\text { Gruppo Controllo }(n= \\
7 \text { ) }\end{array}$} & \multicolumn{2}{|c|}{$\begin{array}{l}\text { Gruppo di Lavoro }(n= \\
20)\end{array}$} \\
\hline & $M$ & $D S$ & $M$ & $D S$ \\
\hline $\begin{array}{l}\text { Media } \\
\text { incontri }\end{array}$ & 179,00 & 51,22 & 185,30 & 42,80 \\
\hline $\begin{array}{l}\text { Media } \\
\text { incontri }\end{array}$ & 154,29 & 36,02 & 179,15 & 35,31 \\
\hline DS pre incontri & 84,43 & 33,20 & 85,20 & 24,21 \\
\hline $\begin{array}{l}\text { DS post incontri } \\
\text { Ipoglicemie }\end{array}$ & 68,14 & 20,95 & 80,65 & 18,11 \\
\hline $\begin{array}{l}\text { rilevate pre } \\
\text { incontri }\end{array}$ & 9,57 & 5,62 & 10,00 & 9,11 \\
\hline $\begin{array}{l}\text { Ipoglicemie } \\
\text { rilevate post } \\
\text { incontri }\end{array}$ & 12,14 & 7,99 & 19,35 & 18,14 \\
\hline
\end{tabular}

\section{Obiettivi specifici di comportamento}

Come riportato in Tabella 6, la maggior parte dei ragazzi del gruppo di lavoro ha raggiunto gli obiettivi generali di gestione promossi durante il percorso. In particolare il $94 \%$ dei pazienti ha introdotto (attivando o modificando) comportamenti adeguati di gestione delle ipoglicemie, il 63\% è in grado di applicare le regole più generali di correzione (regola del 15). Le indicazione di best practice sono state applicate in relazione all'autoverifica delle lipodistrofie, alla rotazione dei siti di iniezione e all'azione di sostituzione dell'ago ad ogni iniezione. L'obiettivo della metodica relativa alla "conta dei carboidrati", in relazione alla applicazione di un calcolo del contenuto 
di carboidrati negli alimenti per gli adeguamenti di terapia, non è stato invece raggiunto.

Tab 6. percentuale di raggiungimento degli obiettivi di comportamento di autogestione specifici promossi misurati attraverso autovalutazione e valutazione con simulate.

\begin{tabular}{|l|l|l|}
\hline & $\begin{array}{l}\text { Comportamento } \\
\text { adeguato } \\
\text { obiettivo } \\
\text { raggiunto } \\
\text { (in percentuale) }\end{array}$ & $\begin{array}{l}\text { Obiettivo } \\
\text { raggiunto in parte } \\
\text { (in percentuale) }\end{array}$ \\
\hline $\begin{array}{l}\text { Presenza del "Kit ipoglicemie" } \\
\text { (presenza di un adeguato } \\
\text { apporto di carboidrati da usare } \\
\text { in caso di ipoglicemia) }\end{array}$ & 94 & 26 \\
\hline $\begin{array}{l}\text { Applicazione regola del 15 } \\
\text { Applicazione calcolo riduzione } \\
\text { eccedenza glicemica }\end{array}$ & 63 & 42 \\
\hline $\begin{array}{l}\text { Utilizzo dello strumento di } \\
\text { "Conta dei carboidrati" }\end{array}$ & 15 & \\
\hline Autoverifica lipodistrofie & 78 & \\
\hline Rotazione dei siti di iniezione & 78 & \\
\hline $\begin{array}{l}\text { Sostituzione ago ad ogni } \\
\text { iniezione }\end{array}$ & 73 & \\
\hline $\begin{array}{l}\text { Riduzione lunghezza ago mm } \\
\text { (da 8 a 5) }\end{array}$ & 94 & \\
\hline
\end{tabular}

La valutazione soggettiva del nuovo modello proposto Analisi della percezione soggettiva del cambiamento

\section{Il punto di vista dei pazienti}

I pazienti del gruppo di lavoro hanno dichiarato un alto livello di gradimento in relazione alla nuova proposta. Il $78 \%$ dei partecipanti dichiara di preferire gli incontri di gruppo, e manifesta piacere e interesse per la tipologia di incontri proposti $(75 \%$ molto $\mathrm{e}$ moltissimo). L'incontro con altri pazienti diabetici è risultato gradito: il 
$66 \%$ ha avuto moltissimo o molto piacere di incontrare altri diabetici e il $75 \%$ ha trovato facile parlare di diabete nei gruppi. Rispetto alla visita ambulatoriale, le attività degli incontri di gruppo sono state valutate più positive e preferibili alla visita individuale. Negli incontri di gruppo "si fanno più cose" (60\%), "si parla di più" (75\%) e "si affrontano le cose in modo diverso" (60\%).

\section{Il punto di vista degli operatori}

Anche i 6 operatori coinvolti nel progetto, che si sono alternati nella conduzione dei gruppi, sono stati invitati a riflettere sul cambiamento proposto e sugli aspetti salienti del nuovo modo di proporsi per la cura. Attraverso la somministrazione di un questionario appositamente costruito che prevedeva risposte multiple sono stati invitati a riflettere sull'efficacia del nuovo approccio proposto e sulle nuove modalità di lavoro attivate. Gli operatori hanno considerato il nuovo approccio al paziente come un modo di lavorare più complesso (4 su 6), ma al tempo stesso più efficace (6 su 6). In particolare, tutti gli operatori hanno descritto come ostacolo maggiore la fatica di essere flessibili sui bisogni di più persone e su più bisogni contemporaneamente. Per quanto riguarda poi il fatto di lavorare in équipe sia nella programmazione degli incontri, che nella gestione, il coinvolgimento in un gruppo di lavoro è stato definito fondamentale ( $5 \mathrm{su} 6)$, ma anche faticoso ( 2 operatori). Nei commenti liberi, sono state evidenziate le fatiche relative all'omogeneizzazione dei metodi di lavoro e al trovare obiettivi in comune.

\section{Discussione}

Come precedentemente affermato, il maggior problema nell'analisi statistica dei dati riguarda l'esiguo numero di partecipanti nel gruppo di controllo che si sono presentati per le visite annue ambulatoriali e per lo scarico dati e utilizzabili quindi nelle rilevazioni di follow up. Tale dato di drop out elevato potrebbe essere causa di alcune differenze non significative tra i due gruppi e sarà uno degli elementi prioritari su cui lavorare nel breve termine.

I dati di osservazione del benessere soggettivo e relativi alla presenza di sintomi o disagi connessi alla malattia cronica, descritti con il termine di burnout, mettono in luce una significativa problematica nella gestione del diabete nel nostro gruppo di lavoro, confermata dai 
dati clinici della emoglobina glicosilata e dal numero ridotto di visite annue effettuate. La valutazione del burnout da diabete può essere difficile, poiché non è sempre possibile misurare le sensazioni di frustrazioni espresse in relazione alla gestione della malattia. I pazienti stessi possono non essere in grado di riconoscere i propri sentimenti. Il burnout da diabete è un termine mediato dalla terminologia classica dove viene applicato alla sindrome da stress lavorativo. Applicato a una malattia cronica che richiede un alto coinvolgimento nella cura come il diabete, sta ad indicare le caratteristiche di un paziente che manifesta sentimenti di disperazione, senso di impotenza, sensazione che gli sforzi non stiano producendo risultati concreti. Il soggetto in burnout si sente esposto e svuotato, manifesta senso di inadeguatezza, sentimenti di colpa e di fallimento. Può inoltre manifestare irritabilità, ostilità, frustrazione. Tali sentimenti producono demotivazione $\mathrm{e}$ riduzione dei comportamenti di cura producendo un effetto di impotenza appresa (Seligman, 1977). L'elevata percentuale di sintomi da burnout da diabete misurata nel nostro campione può essere spiegata da due fattori: la poca compliance rilevata e il numero di anni di diabete poiché tali pazienti, pur giovani, hanno avuto quasi tutti l'esordio nell'infanzia.

A partire da tali considerazioni si rileva un primo importante cambiamento relativo al numero di visite riferito dai partecipanti al gruppo di lavoro, che è aumentato nel tempo e rispetto al gruppo di controllo. Ciò a indicare che i pazienti coinvolti nel nuovo percorso mostrano un primo livello di gradimento e interesse attraverso la partecipazione continuativa agli incontri. Le assenze ai gruppi sono tra l'altro sempre state giustificate.

Altro dato importante misurato è relativo alla discrepanza tra le competenze del sapere e del saper fare, che indica come il non compenso glicemico rilevato non sia in funzione dei soli bisogni informativi ma riguardi la difficoltà nell'attivare $\mathrm{i}$ comportamenti di automonitoraggio adeguati. Anche il numero di autocontrolli glicemici effettuato dal gruppo di lavoro aumenta nel tempo in modo più pronunciato rispetto al gruppo di controllo e, anche se gli effetti rilevati non sono significativi, i dati mostrano che è importante riprendere a lavorare sulla consapevolezza della funzione di tali azioni per l'autocura. La valutazione degli obiettivi specifici conferma la tendenza a tali modifiche comportamentali verso l'autocura.

Per quanto riguarda la valutazione soggettiva del cambiamento, i partecipanti mostrano di gradire la proposta fatta e di preferirla all'incontro individuale e manifestano piacere e interesse per la 
tipologia di incontri proposti. L'incontro con altri pazienti è stato giudicato gradito e le attività degli incontri di gruppo, messe a confronto con la visita ambulatoriale, sono state valutate da tutti i partecipanti positivamente.

Una delle finalità del percorso promosso dal nuovo progetto riguardava anche l'approccio di cura e lo spazio di azione degli operatori. Infatti era stato pensato e strutturato anche per favorire un nuovo atteggiamento educativo e uno spazio di cambiamento. I risultati mostrano un gradimento generale del nuovo modello proposto; tutti gli operatori coinvolti hanno considerato il nuovo modello educativo e l'applicazione di un approccio biopsicosociale al paziente come un modo di lavorare più complesso ma più efficace. Non mancano di descrivere le fatiche relative al cambiamento loro richiesto, e definiscono come ostacolo maggiore il fatto di lavorare con più persone contemporaneamente ma nel complesso valutano come molto positivo il nuovo approccio. Va poi rilevato che nei commenti liberi, l'efficacia non è stata espressa solo rispetto al fornire cure adeguate in modo diretto ai pazienti, ma anche in funzione del fatto che tale modalità di relazione permette agli operatori di avvicinarsi al mondo del paziente e scoprire con lui cosa gli è utile ai fini della cura, informazioni che spesso non sono disponibili nell'incontro individuale. Anche il nuovo modo di lavorare in équipe è stato valutato positivamente, sia rispetto alla fase di programmazione degli incontri che nella gestione del gruppo di pazienti.

Tutti questi dati confermano lo strumento di empowering di gruppo come un importante mezzo educativo in quanto sembra favorire la compliance e la relazione di cura e di conseguenza rende possibile le azioni di monitoraggio da parte degli operatori, fondamentali per la prevenzione di complicanze da diabete.

\section{Conclusioni}

\section{Promuovere la motivazione degli operatori}

Un elemento rilevato e degno di una piccola nota finale riguarda il rischio di demotivazione degli operatori. Come si evidenzia dai risultati, i cambiamenti nei comportamenti dei pazienti sono lenti e poco evidenti e lo strumento dell'empowering è di per sé considerato, legittimamente, complesso. La lenta progressione dei pazienti, i piccoli passi "stile gambero" che l'approccio promuove, danno frutti sul lungo 
termine. Accettare momenti di regressione e di lento cambiamento fa parte degli interventi di sviluppo delle risorse personali per ottenere un cambiamento profondo. Tale approccio alla cura richiede però un equilibrio e molta determinazione da parte degli operatori coinvolti che devono saper definire e ridefinire il loro obiettivo e prevenire lo scoraggiamento. Devono cioè saper guardare ai loro pazienti come persone, sentirsi di accompagnarli in un processo di evoluzione e crescita; e non considerare i loro progressi come risultato diretto del loro intervento o come misura delle loro prestazioni professionali. Insomma devono passare da un atteggiamento direttivo di cura ad uno di fiducia nelle capacità dell'uomo.

\section{Sviluppi futuri}

La definizione di skills ci parla di abilità, attitudine, destrezza, capacità innata o acquisita nel tempo. Sottolineare questa dimensione del tempo necessario per sviluppare una nuova abilità ci pare l'elemento più importante per definire gli obiettivi futuri. Gli obiettivi principali sono relativi al mantenimento della motivazione alla partecipazione ai gruppi per i pazienti e al mantenimento della motivazione ad utilizzare uno strumento così complesso come è l'empowering per gli operatori.

A livello di ricerca è necessario trovare un modo per invitare i partecipanti del gruppo di controllo ad una valutazione, sia per offrire nuovamente loro la possibilità di inserirsi nei gruppi di lavoro, sia per avere più dati di confronto.

Altro importante obiettivo è quello di mantenere e consolidare i comportamenti di automonitoraggio fondamentali per l'autogestione e l'autocura richiesta. Una proposta a medio termine è legata al sostenere l'equilibrio e il benessere attraverso il promuovere consapevolezza, affinché il diabete entri a far parte della vita dei pazienti non solo come un problema, ma anche come una opportunità per adottare uno stile salutare di vita. Curare la malattia cronica con un approccio globale alla persona significa aiutare il malato a intravedere ciò che sarà, per evitarlo prima che accada. 


\section{Riferimenti bibliografici}

Anderson R.M., Funnell M.M. e Arnold M.S (1996). Using the empowerment approach to help patients change behaviour. In: Anderson B.J. and Rubin R, editors., Practical Psychology for Diabetes Clinicians. Alexandria, VA: American Diabetes Association (trad.it: Psicologia pratica per diabetologi. IPadova 1 Poligrafo, 2002)

Assal J.P. e Lacroix A. (2000). Therapeutic education of patients. Paris: Edition Vigot.

Dallago L. (2006). Che cos'è l'empowerment. Roma: Carocci.

Funnell M.M. e Mensing C.R. (2005). Diabetes education in the management diabetes in complete nurses's guide to diabetes care. American Diabetes Association Inc.

Gentili P. e Gentile L. (2010). Diabete e alessitimia: stato attuale e prospettive. Rassegna. Giornale Italiano di diabetologia e Metabolismo, 30: 65-70.

Green, L.W. e Raeburn J. (1990). Contemporary developments in health promotion: Definitions and challenges. In: Bracht N. (Editor). Health promotion at the community level (pp. 29-44).Newbury Park, CA: Sage.

Lacroix A. e Assal J.P. (2005). Educazione terapeutica dei pazienti. Torino Ed. Minerva Medica.

Marmocchi P., Dall'Aglio C . e Tannini M., (2004). Educare le life skills: come promuovere le abilità psicosociali e affettive secondo l'Organizzazione Mondiale della Sanità. Trento: Erickson

Miselli V. (2006) Qualcosa è cambiato. Qualche domanda e qualche riflessione sull'educazione terapeutica Giornale Italiano di Diabetologia e Metabolismo, 26: 45-47.

Polonsky W.H. (1996) Understanding and treating patients with diabetes burnout. In Anderson B.J., and Rubin R,, eitors, Practical Psychology for Diabetes Clinicians. Alexandria, VA: American Diabetes Association; (trad. it: Psicologia pratica per diabetologi.Padova Il Poligrafo, 2002).

Porta M, e Trento M (2004). Romeo: rethink organization to improve education and outcomes. Diabetic Medicine, 21: 644-645.

Seligman M.E.P. (1977). Helplessness: On Depression, development and death. San Francisco: Freeman.

Vespasiani G., Nicolucci A., Erle E,, Trento M., e Miselli V. (2002). Validazione del Questionario sulla conoscenza del diabete. GISED 2001. Giornale Italiano di Diabetologia e Metabolismo, 22: 109-120.

World Health Organizatio (1992). Skills for life. Geneve: WHO.

Zoffmann V. e Lauritzen T. (2006). Guided self -determination improves life skills with Type 1 diabetes and A1C in randomized controlled trial. Patient Education and Counselling, 64: 78-86. 